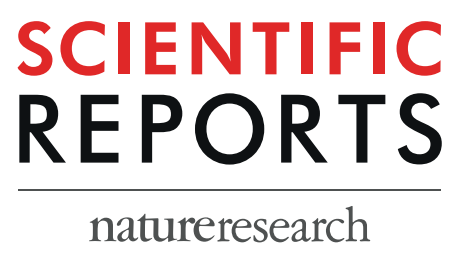

Received: 5 February 2019

Accepted: 8 July 2019

Published online: 31 July 2019

\title{
Comparative Study of
} Subcutaneous and Orthotopic Mouse Models of Prostate Cancer: Vascular Perfusion, Vasculature Density, Hypoxic Burden and BB2rTargeting Efficacy

Wenting Zhang ${ }^{1,2}$, Wei Fan ${ }^{1,2}$, Satyanarayana Rachagani ${ }^{4}$, Zhengyuan Zhou ${ }^{5}$, Subodh M. Lele ${ }^{6}$, Surinder K. Batra ${ }^{3}$ \& Jered C. Garrison ${ }^{1,2,3,4}$

The gastrin-releasing peptide receptor (BB2r) is overexpressed in a variety of cancers including prostate cancer. As a consequence, the development of BB2r-targeted diagnostic/therapeutic radiopharmaceuticals has been widely explored. Both subcutaneous and orthotopic mouse models have been extensively used in BB2r-targeted agent development, but side-by-side studies examining how biological parameters (tumor perfusion efficacy, hypoxic burden and microvasculature density) impact BB2r-targeted agent delivery has not been reported. Herein, we examine these biological parameters using subcutaneous and orthotopic PC-3 xenografts. Using a dual isotope biodistribution study, tumor perfusion was accessed using [ $\left.{ }^{99 \mathrm{~m}} \mathrm{Tc}\right] \mathrm{NaTCO}_{4}$ and $\mathrm{BB} 2 \mathrm{r}$-targeted uptake evaluated by utilization of a novel ${ }^{177}$ Lu-labeled conjugate $\left(\left[{ }^{177}\right.\right.$ Lu $]$ Lu-DOTA-SP714). Immunofluorescence, immunohistochemistry and autoradiography were utilized to examine the tumor vascular density, hypoxic burden and microdistribution of the BB2r-targeted agent. Our studies demonstrated that compared to the subcutaneous model the PC-3 orthotopic tumors had significantly higher levels of perfusion that led to higher BB2r-targeted uptake and lower levels of hypoxia burden. It is anticipated that our results will allow researchers to better understand the biological variables affecting drug delivery and assist them in more clearly interpreting their results in this common prostate cancer mouse model.

The gastrin-releasing peptide receptor (BB2r) is a G-protein coupled receptor that has been of significant interest to the field of cancer drug development due to its overexpression in a variety of cancers, including prostate cancer ${ }^{1}$. Accordingly, numerous theranostic BB2r-targeted agents have been developed over the years with several agents going on to clinical trials ${ }^{2-6}$. Generally, these agents have been based on synthetic derivatives of the C-terminal portions of the bombesin (BBN) peptide, which retains nanomolar affinities for the BB2r. To evaluate newly developed BB2r-targeted agents, researchers have numerous tumor models (e.g., subcutaneous, orthotopic and genetically engineered mouse models) from which to choose $e^{7}$. How well these models mirror the pathology and clinical chemotherapeutic response of human cancers is generally inversely related to the technical difficulties in generating the model. As such, researchers typically rely on relatively simple subcutaneous tumor xenografts to first study the biological performance of new agents. These models are invaluable for examining

${ }^{1}$ Department of Pharmaceutical Sciences, University of Nebraska Medical Center, Omaha, NE, USA. ${ }^{2}$ Center for Drug Delivery and Nanomedicine, University of Nebraska Medical Center, Omaha, NE, USA. ${ }^{3}$ Eppley Cancer Center, University of Nebraska Medical Center, Omaha, NE, USA. 'Department of Biochemistry and Molecular Biology, University of Nebraska Medical Center, Omaha, NE, USA. ${ }^{5}$ Department of Radiology, Duke University Medical Center, Durham, NC, USA. ${ }^{6}$ Department of Pathology and Microbiology, University of Nebraska Medical Center, Omaha, NE, USA. Correspondence and requests for materials should be addressed to J.C.G. (email: jcgarrison@ unmc.edu) 
a

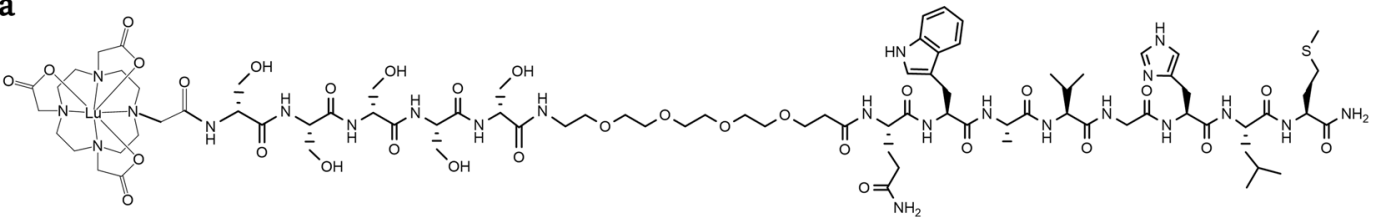

b

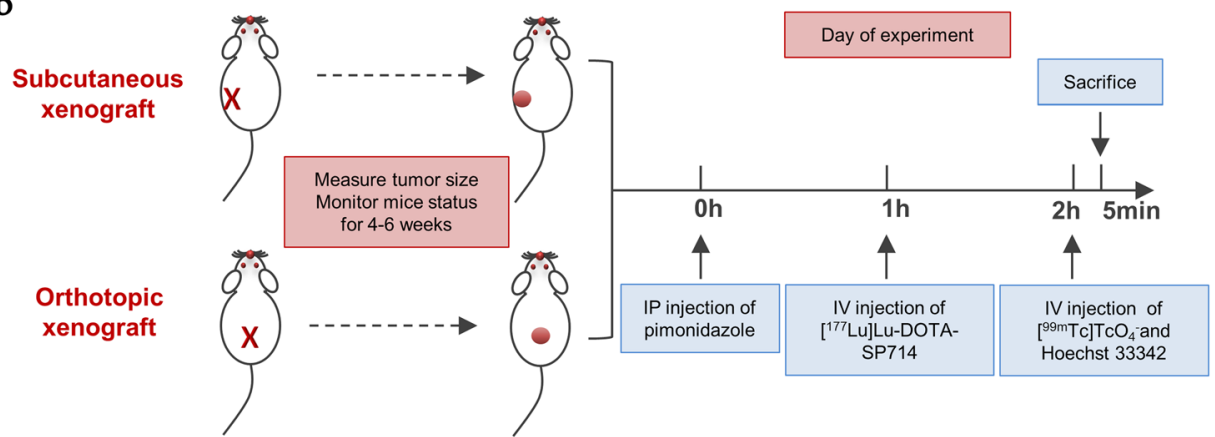

Figure 1. (a) Structure of $\left[{ }^{177} \mathrm{Lu}\right] \mathrm{Lu}-\mathrm{DOTA}-\mathrm{SP} 714$. (b) Experimental design and timeline.

\begin{tabular}{|c|c|c|c|c|c|c|}
\hline \multirow[b]{2}{*}{ Analogue } & \multirow[b]{2}{*}{ Molecular Formula } & \multicolumn{2}{|c|}{$\mathbf{M W}\left(\mathbf{m} / \mathbf{z},[\mathbf{M}+\mathbf{H}]^{+}\right)$} & \multirow{2}{*}{$\begin{array}{l}\text { RP-HPLC Retention } \\
\text { Time/min }\end{array}$} & \multirow[b]{2}{*}{$\mathrm{IC}_{50} / \mathbf{n M}^{\dagger}$} & \multirow[b]{2}{*}{$\operatorname{LogD}\left(\mathrm{pH}_{7.4}\right)^{*}$} \\
\hline & & Calcd & Found & & & \\
\hline DOTA-SP714 & $\mathrm{C}_{85} \mathrm{H}_{137} \mathrm{~N}_{23} \mathrm{O}_{31} \mathrm{~S}$ & 2008.0 & 2008.6 & 8.67 & $9.8 \pm 1.7$ & - \\
\hline Lu-DOTA-SP714 & $\mathrm{C}_{85} \mathrm{H}_{134} \mathrm{LuN}_{23} \mathrm{O}_{31} \mathrm{~S}$ & 2179.9 & 2179.9 & 8.65 & $1.0 \pm 1.7$ & $-2.2 \pm 0.1$ \\
\hline
\end{tabular}

Table 1. Characterization of Conjugate. ${ }^{\dagger}$ Values represent mean $\pm \operatorname{SEM}(n=6) .{ }^{*}$ Values represent $\operatorname{mean} \pm S D$ $(\mathrm{n}=3)$.

the pharmacokinetic profiles of agents, verifying in vivo BB2r-targeting and identifying lead compounds. Yet, it is well-known that the tumor biology of subcutaneous xenografts is not as reflective as other models (e.g., orthotopic) of human tumors ${ }^{8}$. Arguably the most common xenograft model to evaluate BB2r-targeted agents is the murine xenograft model utilizing the human prostate cancer PC-3 cell line, which has been shown to highly overexpress the BB2 $\mathrm{r}^{9-12}$. Previous reports have compared BB2r-targeted uptake in subcutaneous and orthotopic PC-3 mouse models ${ }^{13}$, but a thorough investigation into how the tumor biology of the models affect BB2r-targeted agent delivery remains unreported.

The tumor microenvironment (TME) is a wide-ranging term utilized to describe the complex environment in which tumor exist ${ }^{14}$. In regard to drug delivery, perhaps the most important TME variable is the density and functionality of the tumor vasculature. Tumor perfusion, essentially how well the tumor vasculature distributes blood throughout the tumor, directly influences the delivery of drugs and biologically important nutrients (e.g., oxygen). As a consequence, many solid tumors, including prostate cancer, contain significant regions of hypoxia due to their distorted and insufficiently developed vasculature ${ }^{15}$. Hypoxia is also recognized as a crucial stimulus for tumor angiogenesis, metastasis and, in many cases, aggressiveness ${ }^{16}$.

The purpose of this study is to elucidate the impact of tumor perfusion and vascular density on BB2r-targeted drug delivery and tumor hypoxia. Specifically, we undertake this investigation in subcutaneous and orthotopic PC-3 xenograft mouse models over a spectrum of tumor volumes to explore similarities and differences between the two models. By this study, we hope to give the research community utilizing these models a better understanding of the cause-effect relationship between these variables and their potential impact on drug delivery. To this end, sodium pertechnetate $\left([99 \mathrm{~m} \mathrm{Tc}] \mathrm{NaTcO}_{4}\right)$, a blood perfusion agent ${ }^{17-22}$, was employed to evaluate the PC-3 tumor perfusion efficiency. The functional vasculature density of the tumors was measured using Hoechst staining $^{23}$. A novel BB2r-targeted analog, DOTA-(D)S-(D)S-(D)S-(D)S-(D)S-PEG $3-B B N(7-14) \mathrm{NH}_{2}$ (DOTA-SP714), was utilized to examine in vivo BB2r uptake and tumor distribution (Fig. 1a). Lastly, the hypoxic burden of the PC-3 tumors was measured by pimonidazole staining ${ }^{24}$.

\section{Results}

Synthesis and radiolabeling of DOTA-SP714. DOTA-SP714 was synthesized with a yield of $26.6 \%$ as determined by HPLC-MS. The peptide was labeled with $\mathrm{LuCl}_{3}$ or $\left[{ }^{177} \mathrm{Lu}\right] \mathrm{LuCl}_{3}$ and subsequently purified by RP-HPLC. Analysis of the chromatograms revealed the purity of the radiolabeled peptide was $\geq 95 \%$ with a radiolabeling yield of $90.5 \%$ (Table 1 ).

Distribution coefficient and competitive binding studies. The distribution coefficient (mean \pm SD) at $\mathrm{pH}=7.4\left(\log \mathrm{D}_{7.4}\right)$ for $\left[{ }^{177} \mathrm{Lu}\right] \mathrm{Lu}-\mathrm{DOTA}-\mathrm{SP} 714$ was determined to be $-2.2 \pm 0.1$ by radiometric analysis. The hydrophilic nature of the radioconjugate suggests that cellular internalization should occur only through 
BB2r-mediated endocytosis. Subsequently, competitive binding studies demonstrated that both peptides demonstrated low nanomolar binding affinity, with ${ }^{\text {nat }} \mathrm{Lu}$-DOTA-SP714 $(1.0 \pm 1.7 \mathrm{nM})$ giving a significantly higher affinity relative to the unlabeled conjugate $(9.8 \pm 1.7 \mathrm{nM})$. Both the $\mathrm{IC}_{50}$ and the $\log _{7.4}$ values are provided in Table 1 .

Radiochemical stability studies. To investigate the susceptibility of [ $\left.{ }^{177} \mathrm{Lu}\right] \mathrm{Lu}$-DOTA-SP714 to radiolytic degradation and examine the efficacy of stabilizers, two stabilizing buffers formulated using ascorbic acid with/ without selenomethionine were tested and compared to a control PBS buffer. The results of these studies are depicted in Figure S2. In PBS alone, $39.9 \%$ of a $0.37 \mathrm{MBq} / \mathrm{mL}(100 \mu \mathrm{Ci} / \mathrm{mL})$ solution of $\left[{ }^{177} \mathrm{Lu}\right] \mathrm{Lu}-\mathrm{DOTA}-\mathrm{SP} 714$ degraded over the course of $72 \mathrm{~h}$. As expected, the addition of ascorbic acid $(40 \mathrm{mg} / \mathrm{mL})$ demonstrated substantial improvements in stability with only $17.3 \%$ radiolytic breakdown. However, the combination of ascorbic acid $(40 \mathrm{mg} / \mathrm{mL})$ and selenomethionine $(0.2 \mathrm{mg} / \mathrm{mL})$ gave superior results with only $5.3 \%$ breakdown observed by the $72 \mathrm{~h}$ time point.

Animal model establishment. In this study, subcutaneous and orthotopic xenograft models were generated. For the subcutaneous model, the diameters of the tumors were determined by caliper measurements, while tumor growth in the orthotopic model was monitored weekly after implantation surgery using bioluminescence imaging (Fig. S3a,b). The orthotopic model gave a gradual increase in estimated tumor volumes over the 6-weeks, while the subcutaneous model showed a sharp and rapid increase in tumor size starting at $4^{\text {th }}$-week (Fig. S3c). While the above measurements were used to non-invasively monitor tumor growth, the assignment of the tumor volume in our subsequent studies is based on caliper measurements of the excised tumors. For both measurements, tumor volumes were calculated by using the following formula: Volume $=\left(\right.$ Length $\times$ Width $\left.^{2}\right) / 2$.

BB2r-Targeted peptide uptake and perfusion in PC-3 tumor xenograft models. To examine the in vivo BB2r-targeting efficacy and tumor perfusion of the two mouse models, we performed dual, simultaneous biodistribution studies utilizing the $\left[{ }^{177} \mathrm{Lu}\right] \mathrm{Lu}-\mathrm{DOTA}-\mathrm{SP} 714$ and $\left[{ }^{99 \mathrm{~m}} \mathrm{Tc}\right] \mathrm{NaTcO}$. The $\% \mathrm{ID} / \mathrm{g}$ in each organ and the total excretion (\%ID) of the radioactivity from the mice are presented in Table S1. Overall, the scatter plots of the tumor uptake of $\left[{ }^{177} \mathrm{Lu}\right] \mathrm{Lu}-\mathrm{DOTA}-\mathrm{SP} 714$ and $\left[{ }^{99 \mathrm{~m}} \mathrm{Tc}\right] \mathrm{TcO}_{4}{ }^{-}$against tumor volume in both animal models are depicted in Fig. $2 \mathrm{a}-\mathrm{d}$. There was a significant moderate to strong positive correlation (Table 2), observed between tumor volume and BB2r-uptake $(\% \mathrm{ID} / \mathrm{g})$ of the $\left[{ }^{177} \mathrm{Lu}\right] \mathrm{Lu}-\mathrm{DOTA}-\mathrm{SP} 714(\mathrm{r}=0.55, \mathrm{p}=0.0049$ and $\mathrm{r}=0.73, \mathrm{p}=0.0001)$ as well as tumor perfusion $\left(\left[{ }^{99 \mathrm{~m}} \mathrm{Tc}\right] \mathrm{TcO}_{4}{ }^{-}\right)(\mathrm{r}=0.47, \mathrm{p}=0.021$ and $\mathrm{r}=0.49, \mathrm{p}=0.017)$ in subcutaneous and orthotopic mice models, respectively. Interestingly, the correlation (Figure S4) between tumor perfusion $\left(\left[{ }^{99 \mathrm{~m}} \mathrm{Tc}\right] \mathrm{NaTcO}_{4}\right)$ and BB2r-uptake ([177 $\left.\left.\mathrm{Lu}\right] \mathrm{Lu}-\mathrm{DOTA}-\mathrm{SP} 714\right)$ was weak to moderate for the subcutaneous $(r=0.28, p=0.19)$ and orthotopic $(r=0.66, p=0.0006)$ models, respectively, suggesting tumor size is a better indicator of BB2r-uptake in both models.

Grouping tumor volumes categorically, $<300,300-700$ and $>700 \mathrm{~mm}^{3}$, revealed some interesting trends (Fig. 2e,f). For the orthotopic model, the mean (mean \pm SD) categorical tumor volumes were $215.62 \pm 103.90$, $574.14 \pm 73.89$ and $1052.78 \pm 256.12 \mathrm{~mm}^{3}$ for $<300,300-700$ and $>700 \mathrm{~mm}^{3}$ groups respectively. With respect to the subcutaneous model, the corresponding mean categorical tumor volumes for $<300,300-700$ and $>700 \mathrm{~mm}^{3}$ groups were $169.06 \pm 74.94,491.69 \pm 114.89$ and $1139.25 \pm 378.68 \mathrm{~mm}^{3}$. In the orthotopic model, the average uptake (mean \pm SD) of the $\left[{ }^{177} \mathrm{Lu}\right] \mathrm{Lu}$-DOTA-SP714 was $8.07 \pm 3.23,8.55 \pm 2.65$ and $11.13 \pm 2.88 \% \mathrm{ID} / \mathrm{g}$ for the $<300,300-700$ and $>700 \mathrm{~mm}^{3}$ groups, respectively. By the ANOVA test, while close $(\mathrm{p}=0.061)$, this trend of increased BB2r-uptake with increasing tumor volume did not meet statistical significance given our study size. For the subcutaneous model, the mean uptake of the radioconjugate increased significantly $(\mathrm{p}=0.0014)$ with increasing tumor size from $4.16 \pm 1.74\left(<300 \mathrm{~mm}^{3}\right)$ to $5.35 \pm 1.75\left(300-700 \mathrm{~mm}^{3}\right)$ to $9.44 \pm 3.66\left(>700 \mathrm{~mm}^{3}\right)$ $\% \mathrm{ID} / \mathrm{g}$. Uptake of the perfusion agent in subcutaneous tumors increased from $0.54 \pm 0.32\left(<300 \mathrm{~mm}_{3}\right)$ to $0.68 \pm 0.49\left(300-700 \mathrm{~mm}^{3}\right)$ to $0.86 \pm 0.37\left(>700 \mathrm{~mm}^{3}\right) \% \mathrm{ID} / \mathrm{g}$ as tumor volume increased $(\mathrm{p}=0.15)$. Likewise, the average tumor uptake of the $\left[{ }^{99} \mathrm{~m} \mathrm{Tc}\right] \mathrm{TcO}_{4}{ }^{-}$for the orthotopic model increased when comparing the $<300 \mathrm{~mm}^{3}$ $(2.23 \pm 1.09 \% \mathrm{ID} / \mathrm{g}), 300-700 \mathrm{~mm}^{3}(2.94 \pm 0.76 \% \mathrm{ID} / \mathrm{g})$ and $>700 \mathrm{~mm}^{3}(3.19 \pm 0.66 \% \mathrm{ID} / \mathrm{g})$ groups $(\mathrm{p}=0.078)$.

The most striking differences were observed when comparing the BB2r-mediated uptake and perfusion between models at each tumor volume category. The average uptake of the $\left[{ }^{177} \mathrm{Lu}\right] \mathrm{Lu}$-DOTA-SP714 for the orthotopic model was 1.9, 1.6 and 1.2-fold higher compared to the respective tumor volumes of the subcutaneous model $\left(<300 \mathrm{~mm}^{3}, \mathrm{p}=0.0055 ; 300-700 \mathrm{~mm}^{3}, \mathrm{p}=0.011\right.$; and $>700 \mathrm{~mm}^{3}$ groups, $\left.\mathrm{p}=0.34\right)$. Similarly, a substantial and statistically significant increase in tumor perfusion was observed for the orthotopic versus the subcutaneous model for each tumor volume category. The average uptake of perfusion tracer in the orthotopic model was 4.1, 4.3 and 3.7-fold higher compared to the respective subcutaneous model $\left(<300 \mathrm{~mm}^{3}, \mathrm{p}=0.0008 ; 300-700 \mathrm{~mm}^{3}\right.$, $\mathrm{p}=0.0001$; and $\left.>700 \mathrm{~mm}^{3}, \mathrm{p}=0.0001\right)$. Based on the examination of the biodistribution data, this large difference in perfusion between models may well be overstated due to a decreased clearance rate of the radiotracer from the orthotopic model. Biodistribution data (Table S1) show that the blood retention for the orthotopic model was 1.5-3.1 times higher compared to the subcutaneous model. Furthermore, this trend was reflected in the well-known stomach uptake of ${ }^{99 \mathrm{~m}} \mathrm{Tc}$-pertechnetate which was approximately 8 -fold higher for the orthotopic

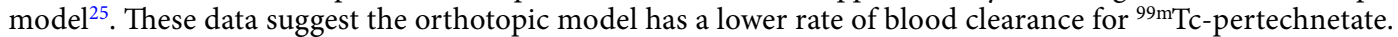
Speculatively, this may be due to the placement of the orthotopic tumor restricting renal excretion ${ }^{26}$ and reducing ${ }^{99 \mathrm{~m}} \mathrm{Tc}$-pertechnetate clearance from the body. If so, this effect was asymmetric since the blood retention values for the BB2r-targeted agent $\left(\left[{ }^{177} \mathrm{Lu}\right] \mathrm{Lu}-\mathrm{DOTA}-\mathrm{SP} 714\right)$ between the two models were not statistically different.

Quantification of hypoxic burden and blood vessel density in PC-3 tumors. Prior to the sacrifice of the mice, pimonidazole (hypoxia marker) and Hoechst (functional vasculature marker), were administered to examine the hypoxia burden and vascular density of the human PC-3 tumor xenografts. The excised human PC-3 tumors were sectioned and histologically evaluated for fluorescence signals using adjacent tumor slices. Both 
a

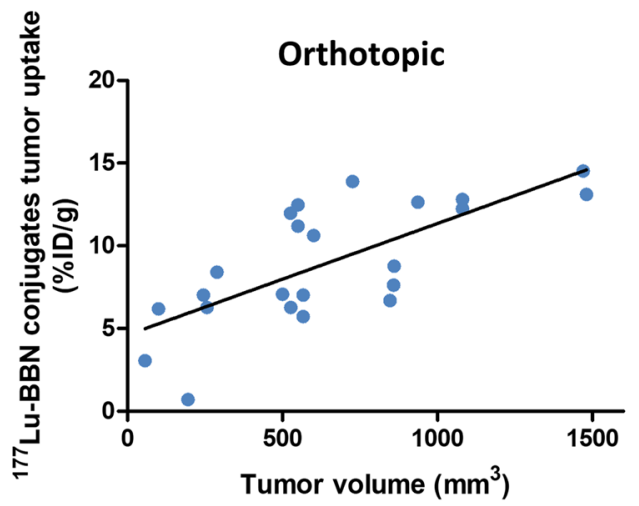

c
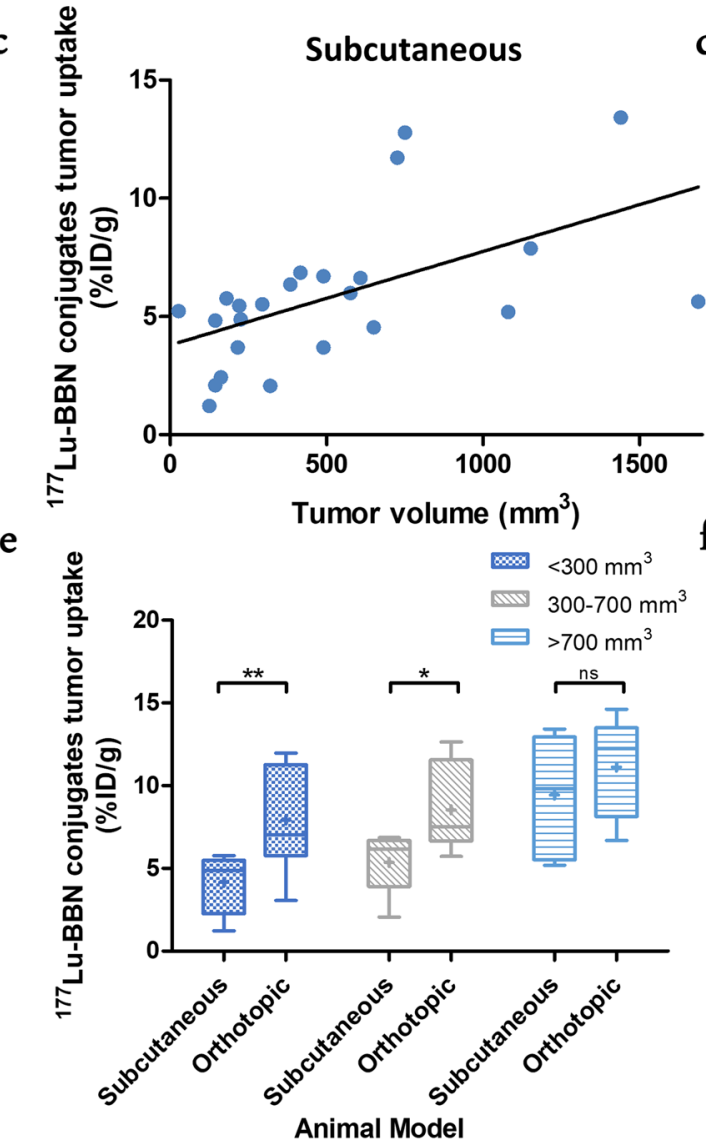

b
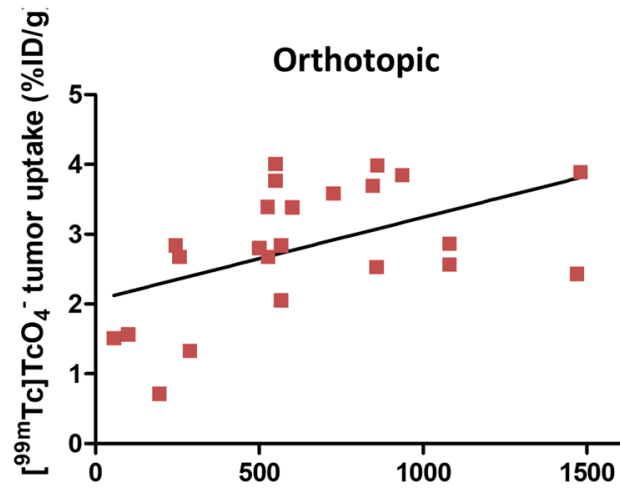

d ธิ

Tumor volume $\left(\mathrm{mm}^{3}\right)$

Subcutaneous

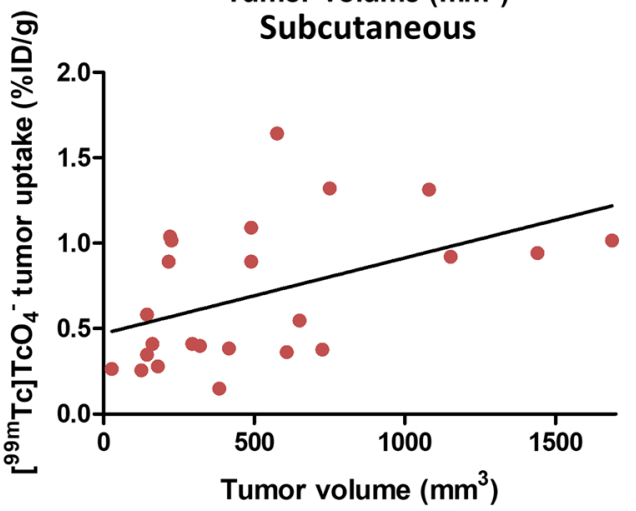

f

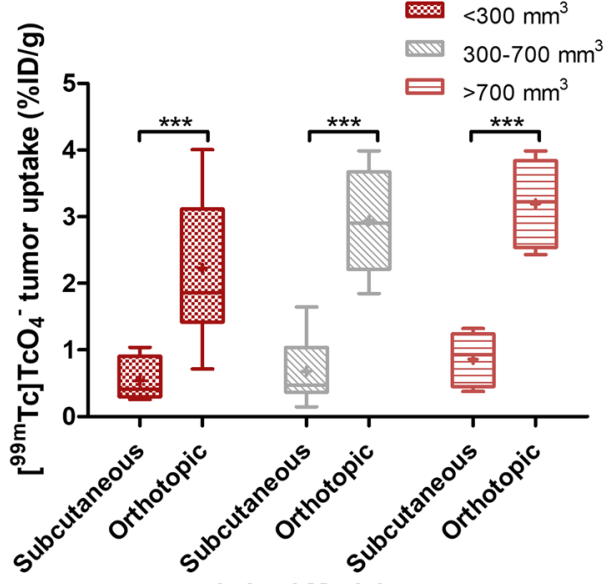

Animal Model

Figure 2. Correlation of tumor uptake of $\left[{ }^{177} \mathrm{Lu}\right] \mathrm{Lu}-\mathrm{DOTA}-\mathrm{SP} 714(\mathbf{a}, \mathbf{c})$ and of $\left[{ }^{99 \mathrm{~m}} \mathrm{Tc}\right] \mathrm{TcO}_{4}{ }^{-}(\mathbf{b}, \mathbf{d})$ in orthotopic $(\mathrm{n}=23)$ and subcutaneous $(\mathrm{n}=24)$ model respectively. $(\mathbf{e}, \mathbf{f})$. Grouped box-whisker plot of tumor uptake of $\left[{ }^{177} \mathrm{Lu}\right] \mathrm{Lu}-\mathrm{DOTA}-\mathrm{SP} 714$ and $\left[{ }^{99 \mathrm{~m} T c}\right] \mathrm{TcO}_{4}{ }^{-}$in two animal models. $(* \mathrm{p}<0.05, * * \mathrm{p}<0.01, * * * \mathrm{p}<0.001$, ns $=$ no significance, + : mean, line at median).

\begin{tabular}{|c|c|c|c|}
\hline \multirow{2}{*}{\multicolumn{2}{|c|}{ Tumor uptake (\%ID/g) }} & \multicolumn{2}{|c|}{ Tumor volume $\left(\mathrm{mm}^{3}\right)$} \\
\hline & & \multirow{2}{*}{\begin{tabular}{|l|} 
Pearson $\mathbf{r}$ \\
0.73 \\
\end{tabular}} & \multirow{2}{*}{\begin{tabular}{|l|}
$\mathbf{p}$-value \\
0.0001
\end{tabular}} \\
\hline \multirow{2}{*}{ Orthotopic } & {$\left[{ }^{[177} \mathrm{Lu}\right] \mathrm{Lu}-\mathrm{DOTA}-S P 714$} & & \\
\hline & {$\left[{ }^{99 \mathrm{~m}} \mathrm{Tc}_{\mathrm{TcO}} \mathrm{Tc}_{4}^{-}\right.$} & 0.49 & 0.017 \\
\hline \multirow{2}{*}{ Subcutaneous } & {$\left[{ }^{177} \mathrm{Lu}\right] \mathrm{Lu}-\mathrm{DOTA}-\mathrm{SP} 714$} & 0.55 & 0.0049 \\
\hline & {$\left[{ }^{99 \mathrm{~m}} \mathrm{Tc}\right] \mathrm{TcO}_{4}^{-}$} & 0.47 & 0.021 \\
\hline
\end{tabular}

Table 2. Pearson Correlation Coefficient and p-Value of Tumor Volume with Uptake of Radiotracer in Mice Models. 


\begin{tabular}{|l|l|l|l|l|}
\hline \multirow{2}{*}{ Tumor volume $\left(\mathbf{m m}^{3}\right)$} & \multicolumn{4}{|l|}{ Mean Intensity $/ \boldsymbol{\mu m}^{2}(\mathbf{m e a n} \pm \mathbf{S E M})^{*}$} \\
\cline { 2 - 5 } & Subcutaneous & Orthotopic \\
\hline$<\mathbf{3 0 0}$ & Hypoxic burden & Vasculature Density & Hypoxic burden & Vasculature Density \\
\hline $\mathbf{3 0 0}-\mathbf{7 0 0}$ & $9.47 \mathrm{e}-6 \pm 1.74 \mathrm{e}-6$ & $4.06 \mathrm{e}-6 \pm 8.54 \mathrm{e}-7$ & $4.33 \mathrm{e}-6 \pm 1.41 \mathrm{e}-6$ & $8.33 \mathrm{e}-6 \pm 1.42 \mathrm{e}-6$ \\
\hline$>\mathbf{7 0 0}$ & $3.84 \mathrm{e}-5 \pm 6.98 \mathrm{e}-6$ & $9.90 \mathrm{e}-6 \pm 1.88 \mathrm{e}-6$ & $1.18 \mathrm{e}-5 \pm 2.64 \mathrm{e}-6$ & $8.67 \mathrm{e}-6 \pm 6.44 \mathrm{e}-7$ \\
\hline
\end{tabular}

Table 3. Mean Intensity/ $\mu \mathrm{m}^{2}$ of Hypoxic Burden and Blood Vessel Density in Tumor Xenograft. *Values represent mean $\pm \operatorname{SEM}(n \geq 6)$.
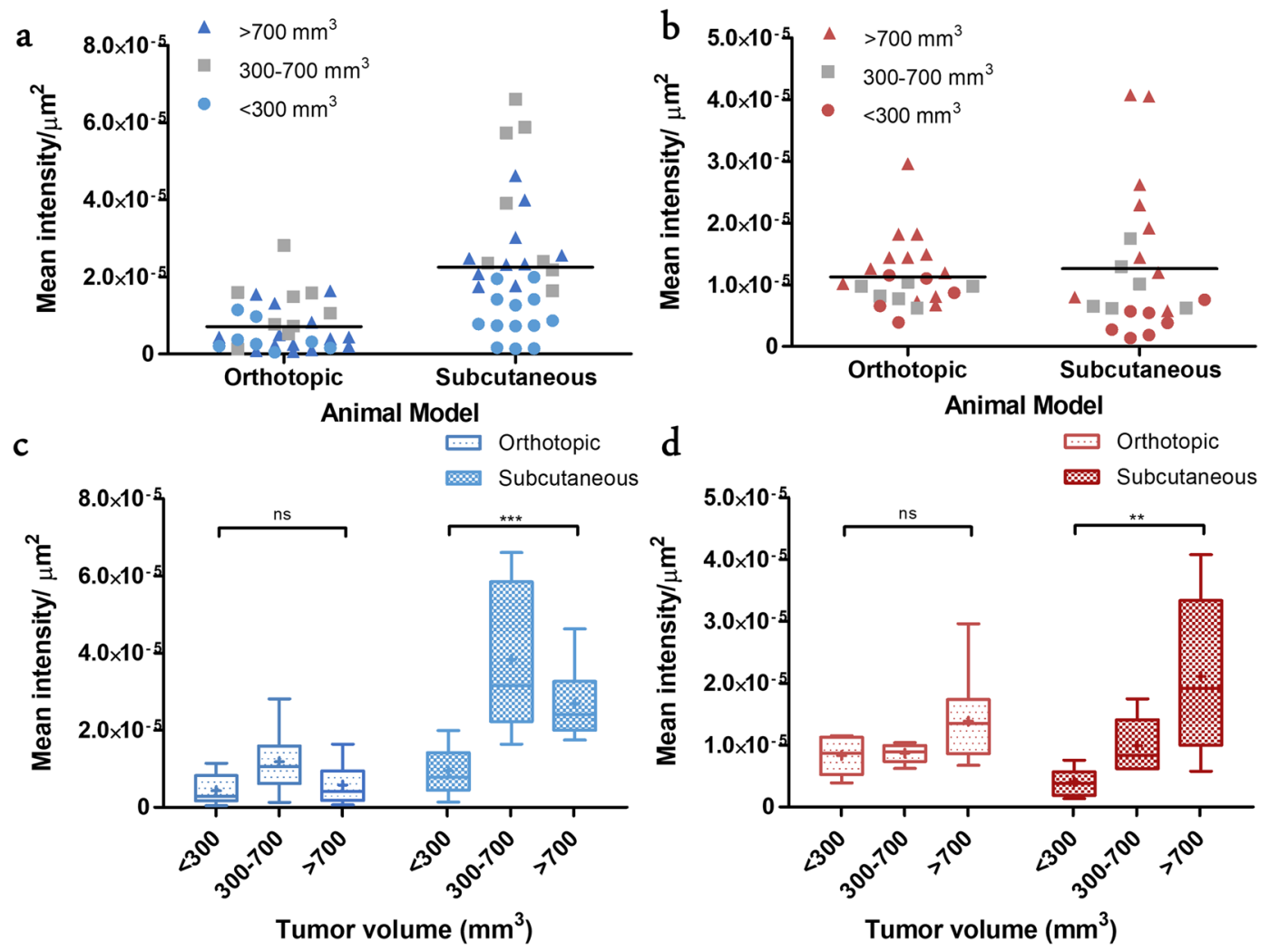

Figure 3. Summary of quantification of (a) hypoxia burden (b) blood vessel density of tumor slides in two mouse models. Line at grand mean. Box-whisker plots are indicating the variance of (c) hypoxia burden and (d) blood vessel density between different tumor volume groups in two animal models. ( + : mean, line at median).

signals were calculated by mean intensity per $\mu \mathrm{m}^{2}$ and grouped by tumor volume groups for each mouse model (Table 3).

On average, subcutaneous tumors exhibited a 3.4-fold higher level of hypoxia burden (signal) than the orthotopic tumors (Fig. 3a). For both xenograft models, the tumors demonstrated a trend toward maximal hypoxia burden for the $300-700 \mathrm{~mm}^{3}$ group (Fig. 3c). Overall, there was no significant difference $(\mathrm{p}=0.13)$ in the hypoxia burden among the tumor volume groups in the orthotopic model (one-way ANOVA). However, a significant difference in hypoxia levels was observed within the tumor volume groups of the subcutaneous mouse model $(\mathrm{p}<0.001)$. A comparison of hypoxia burden for the $<300 \mathrm{~mm}^{3}$ tumor groups revealed no significant difference $(p=0.053)$ between models. In contrast, significant differences $(p=0.002$ and $p<0.0001$, correspondingly) between xenograft models were observed for the 300-700 and $>700 \mathrm{~mm}^{3}$ groups, largely due to the substantial increase in hypoxia levels in the subcutaneous models for tumor volumes exceeding $300 \mathrm{~mm}^{3}$. Specifically, the hypoxia burden increased 4.0 -fold in the subcutaneous model for the $300-700 \mathrm{~mm}^{3}$ group compared to the $<300 \mathrm{~mm}^{3}$ group $(\mathrm{p}<0.001)$. In both tumor models, the hypoxia levels decreased on average by $30-50 \%$ when the tumor size exceeded $700 \mathrm{~mm}^{3}$ compared to the respective $300-700 \mathrm{~mm}^{3}$ group $(\mathrm{p}>0.05)$.

The functional tumor blood vessel density of the excised PC-3 tumors are depicted in Fig. 3b. On average, the subcutaneous tumors had a slightly higher functional vascular density compared to the orthotopic tumors. For both xenograft models, there was a trend toward higher vascular density as tumor volume increased (Fig. 3d). For the subcutaneous model, there was a 2.4 and 5.2-fold increase of functional vascular density in the 300-700 $(\mathrm{p}=0.013)$ and $>700 \mathrm{~mm}^{3}$ groups $(\mathrm{p}=0.0041)$ relative to the $<300 \mathrm{~mm}^{3}$ group. With respect to the orthotopic 
Animal Model
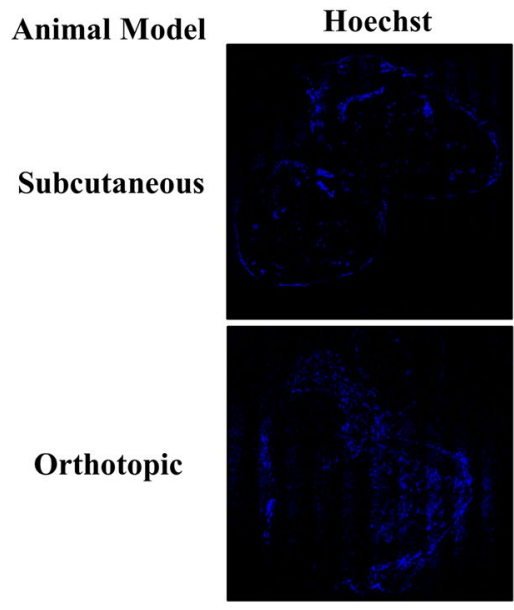

Subcutaneous

Orthotopic

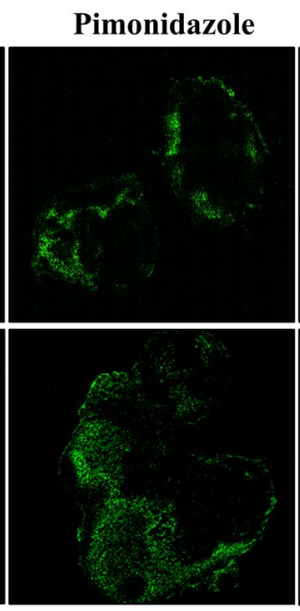

Autoradiography

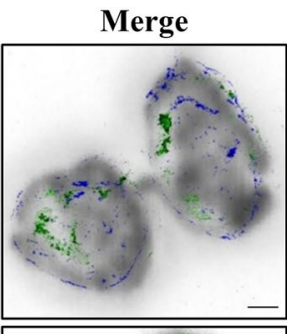

Figure 4. Representative confocal microscopy images of adjacent tumor slides stained with Hoechst 33342 (blue) and anti-pimonidazole-FITC (green) and performed autoradiography respectively in two mouse models. Scale bar: $1 \mathrm{~mm}$.

tumors, no increase in vascular density was observed for the $300-700 \mathrm{~mm}^{3}(\mathrm{p}=0.82)$ group compared to the $<300 \mathrm{~mm}^{3}$ group. Although, a 1.7 -fold increase in vessel density was observed for the $>700 \mathrm{~mm}^{3}(\mathrm{p}=0.084)$ tumor volume group relative to the smaller groups. For tumor group comparisons between models, the orthotopic tumors of the $<300 \mathrm{~mm}^{3}$ group had a significantly $(\mathrm{p}=0.0021)$ higher vascular density compared to the subcutaneous model, but no statistically significant differences were found between the $300-700$ and $>700 \mathrm{~mm}^{3}$ groups ( $\mathrm{p}=0.55$ and $\mathrm{p}=0.11$, correspondingly).

Qualitative examination of the distribution of the BB2r-Targeted peptide in tumor xenografts. To better examine the distribution of [ $\left.{ }^{177} \mathrm{Lu}\right] \mathrm{Lu}-\mathrm{DOTA}-\mathrm{SP} 714$ in the two xenografts models in relation to the hypoxic fractions and functional vasculature of tumors, sections of tumors were examined by autoradiography (BB2r-targeted peptide) and confocal microscopy to determine the hypoxic areas (Green) and functional vasculature (Blue). The representative images from the $300-700 \mathrm{~mm}^{3}$ group are shown in Fig. 4 . As expected, the predominant localization of the $\left[{ }^{177} \mathrm{Lu}\right] \mathrm{Lu}-\mathrm{DOTA}-\mathrm{SP} 714$ was in areas that had significant functional vasculature. While in some areas that lacked vasculature were hypoxic and exhibited a significantly lower concentration of $\left[{ }^{177} \mathrm{Lu}\right] \mathrm{Lu}-\mathrm{DOTA}-\mathrm{SP} 714$.

H\&E staining of non-target tissues. Upon dissection of the mice from the orthotopic mouse model with tumors exceeding $700 \mathrm{~mm}^{3}$, gross anatomy abnormalities of the kidneys, pancreas and liver were perceived. The kidneys were often found to be pale and enlarged and the formation of fluid-filled cysts on the surface of the kidneys was observed, likely due to hydronephrosis. In several cases, enlargement and/or discoloration of the liver and pancreas were also noted. The tissues taken from orthotopic models along with an analogous subcutaneous model $\left(>700 \mathrm{~mm}^{3}\right.$ group) and normal control mouse were sectioned and underwent H\&E staining, see Fig. 5. All of the sectioned tissues associated with the orthotopic model were more diffuse than subcutaneous and normal controls probably due to the increased interstitial volume resulting from reduced urine output. For the orthotopic tumor group, micrometastases were observed in the pancreas, but, surprisingly, were not found in the liver or kidney sections. No micrometastases were observed in the sections obtained from the subcutaneous model.

\section{Discussion}

The purpose of this work is to investigate how the vascular density and perfusion of tumors impact the hypoxia burden and drug delivery of BB2r-targeted agents. Specifically, we investigated these factors in subcutaneous and orthotopic xenografts across a range of tumor volumes. By understanding this, we hope to provide researchers a better understanding of these biological variables as well as the advantages and limitations of both subcutaneous and orthotopic models for BB2r-targeted agent development.

We first synthesized and characterized a new hydrophilic, BB2r-targeted radioconjugate $\left(\left[{ }^{177} \mathrm{Lu}\right]\right.$ Lu-DOTA-SP714). In addition to examining it's chemical and in vitro properties, we also accessed the solution stability of the radioconjugate. This investigation was prompted out of the need to perform in vivo experiments with large numbers of mice and the desire to reduce the need for the constant synthesis of the radioconjugate. It is well established that ionizing radiation (e.g., $\alpha^{2+}$ and $\beta^{-}$particles) can directly or indirectly, through the generation of solvated electrons and free radicals species, degrade peptides and proteins ${ }^{27}$. Ascorbic acid and selenomethionine have been demonstrated to have a protective effect against radiolytic degradation in both preclinical and clinical studies ${ }^{28-30}$. Indeed, Chen and co-workers examined these radioprotectants among others with $\left[{ }^{177} \mathrm{Lu}\right]$ Lu-AMBA, a clinically investigated BB2r-targeted agent ${ }^{31}$. Similar to their findings, our studies revealed that the combination of the two radioprotectants, ascorbic acid $(40 \mathrm{mg} / \mathrm{mL})$ and selenomethionine $(0.2 \mathrm{mg} / \mathrm{mL})$, was better than ascorbic acid alone with approximately $95 \%$ of the radioconjugate intact by $72 \mathrm{~h}$. 


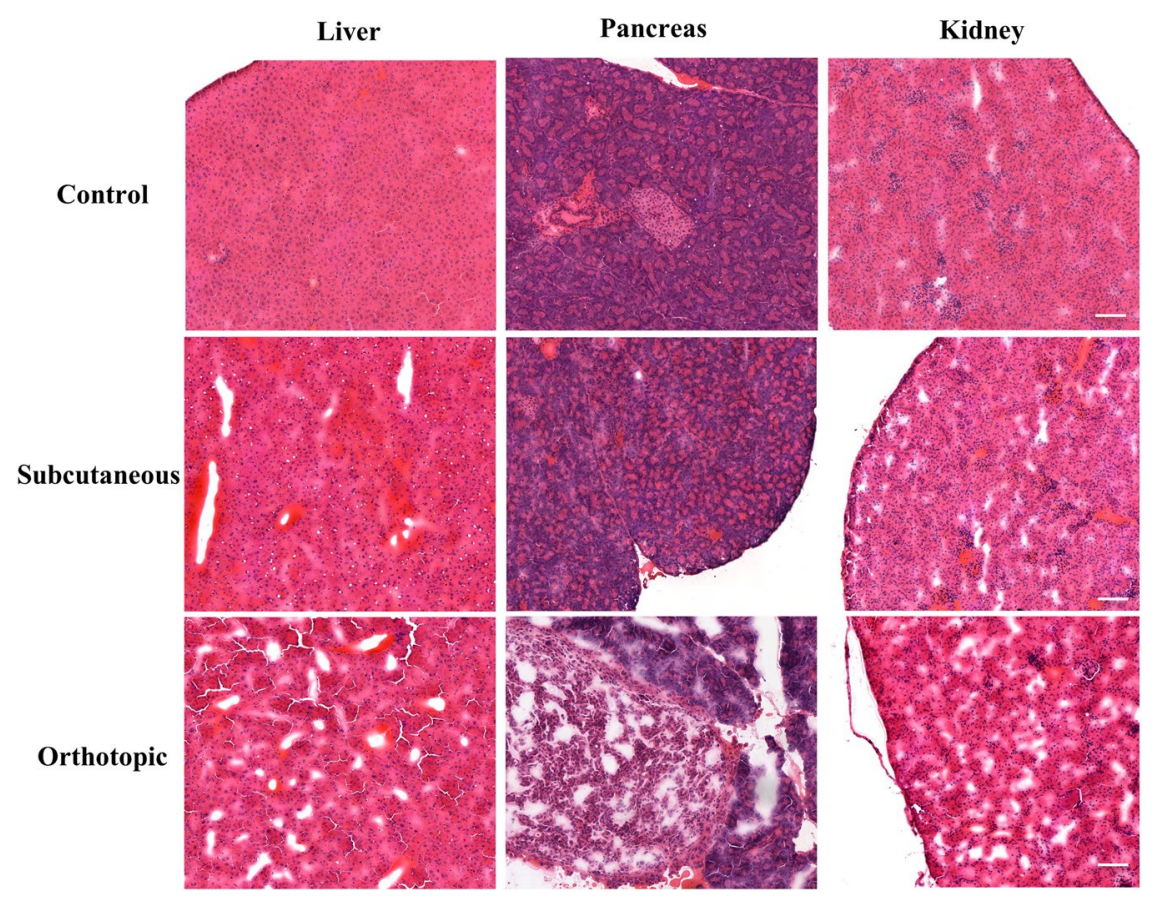

Figure 5. Representative $H \& E$ image of the liver, pancreas and kidney tissue sections $(10 \times)$ of non-tumor bearing, subcutaneous and orthotopic xenograft mouse respectively. Scale bar: $100 \mu \mathrm{m}$.

The establishment of the subcutaneous and orthotopic PC-3 xenograft model was carried out over a four to eight-week period depending on the model and the desired tumor size. The measured tumor size for orthotopic tumors initially outpaced subcutaneous tumors, but the subcutaneous tumors demonstrated a more rapid growth at approximately 4 -weeks post-inoculation. The disparity of growth rates between two models likely stem from the different measurement techniques employed and the initial cell numbers administered. Based on body weight measurements, the overall health of the mice appeared good across models and tumor size ranges investigated (Figure S5). However, for the largest orthotopic tumor volume group $\left(>700 \mathrm{~mm}^{3}\right)$, abnormalities were found upon gross examination of the kidneys, liver and pancreas. This prompted the histological examination of these tissues for both models. The tissues obtained from the orthotopic model were more diffuse and were consistent with larger interstitial water content, likely due to urinary obstruction resulting from the PC-3 tumor in the prostate. This observed condition has been noted for other orthotopic prostate cancer mouse models ${ }^{32}$.

The examination of the perfusion characteristics of the two models revealed some interesting findings. The average perfusion of the tumors increased as tumor size increased. However, the orthotopic model showed substantially higher tumor perfusion (3.7-4.3 fold) compared to the subcutaneous model. This is interesting given the similar amount of functional vasculature in both models based on Hoechst staining. This comparably higher perfusion rate has also been observed in other orthotopic relative to subcutaneous models ${ }^{33-35}$. A factor that may be contributing to the lower perfusion of subcutaneous tumors is a higher interstitial fluid pressure (IFP). In general, the functional vasculature of the orthotopic tumors is more spatially distributed and have larger vessel diameters relative to subcutaneous tumors ${ }^{36,37}$. These factors have been shown to reduce the level of IFP. With that said, as we discussed earlier, the perfusion agent ${ }^{99 \mathrm{~m}} \mathrm{Tc}$-pertechnetate) exhibited a lower rate of renal clearance possibly overstating the magnitude in the differences in perfusion between the two models. Careful consideration should be given regarding the blood and renal clearance of the radiotracer when comparing tumor uptake values.

The BB2r-targeting efficacy of the $\left[{ }^{177} \mathrm{Lu}\right] \mathrm{Lu}-\mathrm{DOTA}-\mathrm{SP} 714$ was examined in both PC-3 xenograft models. Generally, the average \%ID/g uptake of the radioconjugate increased as the tumor size increased. Specifically, orthotopic tumors exhibited significantly higher uptake compared to subcutaneous tumors across tumor groups. This is almost certainly due to the increased perfusion of orthotopic over subcutaneous tumors.

Using pimonidazole, the hypoxia burden of the tumors was evaluated for both models. For the orthotopic tumors, no significant differences in hypoxia levels were observed across the tumor volume ranges. However, the subcutaneous tumors exhibited significant increases in hypoxia with tumor sizes that exceeded $300 \mathrm{~mm}^{3}$. The increased hypoxia levels for the 300-700 and $>700 \mathrm{~mm}^{3}$ tumor groups may be attributable to the spatial distribution of the functional vascular on the periphery of tumors. For small tumors, the peripheral vasculature can adequately perfuse the whole tumor. However, as the tumor grows vasculature may become incapable of perfusing the center of the tumor resulting in significant increases in hypoxia burden ${ }^{38}$ and tumor necroses ${ }^{39-42}$. While hypoxia was observed for both models, medium to large subcutaneous tumors more reliably gave tumors with significant fractions of hypoxia. This phenomenon has been observed by other researchers as well ${ }^{43,44}$. In this particular study, we did not examine the extent of tumor necroses and its correlation to PC-3 tumor volumes to determine its impact, if any, on BB2r-targeted agent delivery. This is certainly an interesting question and 
something we may examine in our future work. Overall, these findings suggest PC-3 tumors $>300 \mathrm{~mm}^{3}$ may be a more appropriate volume to investigate the efficacy of hypoxia-targeted or hypoxia-selective agents.

\section{Conclusion}

Orthotopic models are known to better simulate clinical prostate cancer, particularly with respect to the tumor microenvironment, compared to subcutaneous models. To better understand how the biology of these tumor models' impact BB2r-targeted agent delivery, we examined the tumor vascular perfusion, microvasculature density and hypoxia burden of orthotopic and subcutaneous PC-3 xenograft mouse models. Compared to the subcutaneous model, the results demonstrate that the orthotopic PC-3 tumors have higher vascular perfusion that leads to higher BB2r targeting as well as a lower hypoxic burden. While the vessel density was slightly lower in the orthotopic model compared to subcutaneous ones, no statistical significance was observed. In general, for both models, BB2r-targeting, perfusion and vascular density increased with increasing tumor volume. Immunofluorescence and autoradiography illustrated the microdistribution pattern of the BB2r-targeted conjugate relative to functional vasculature and hypoxic regions. As expected, higher concentrations of the radiolabeled conjugate were observed near functional vasculature compared to hypoxic regions that were devoid of functional vasculature. Overall, this work demonstrates that the tumor microenvironments of orthotopic and subcutaneous PC-3 tumors are impactfully different in terms of drug delivery. Careful consideration should be taken when comparing the data of BB2r-targeted agents, as well as other targeted agents, in orthotopic and subcutaneous tumor models.

\section{Materials and Methods}

Full details regarding the materials and equipment are presented in the supplemental materials. Also given in the supporting information is our methodology concerning peptide synthesis, cell culture, luciferase transfection, distribution coefficient studies and competitive binding studies.

Radiochemical stability studies. The radiochemical stability of [ $\left.{ }^{177} \mathrm{Lu}\right] \mathrm{Lu}-\mathrm{DOTA}-\mathrm{SP} 714$ was evaluated by RP-HPLC using various stabilizing buffers. Briefly, $0.37 \mathrm{MBq}$ of radiolabeled compounds were incubated with $1 \mathrm{~mL}$ of: A) PBS; B) ascorbic acid in $0.9 \%$ sodium chloride solution $(40 \mathrm{mg} / \mathrm{ml})$; and C) Selenomethionine $(0.2 \mathrm{mg} / \mathrm{ml})$ and ascorbic acid $(40 \mathrm{mg} / \mathrm{ml})$ in $0.9 \%$ sodium chloride solution at $4{ }^{\circ} \mathrm{C}$ for 24,48 and $72 \mathrm{~h}$. The stability of the radioconjugate at each time point was calculated based on the integration of the RP-HPLC chromatograms.

Animal models. All animal experiments were performed in accordance with the NIH animal use guideline and protocol approved by the Institutional Animal Care and Use Committee (IACUC) at the UNMC. NOD. CB17-Prkdc sicd IJ (NOD SCID) mice were purchased from the Jackson Laboratory (Bar Harbor, ME). The mice were housed in groups of five in the UNMC animal facility for the entire tumor generation period. All mice were under constant temperature (set up at $21^{\circ} \mathrm{C}$ ) and humidity on a 12-hour light/dark cycle, which lights on at 7:00 am. Standard food and filtered water were available ad libitum.

For the subcutaneous model ${ }^{9}, 5$-week old female SCID mice were inoculated in the flanks with $5 \times 10^{6} \mathrm{PC}-3$ cells (American Type Culture Collection (ATCC), VA) in Matrigel ${ }^{\circledR}$. The tumor size was monitored by caliper. For the generation of the orthotopic model ${ }^{45}$, the PC-3-Luc cells were selected by $0.5 \mu \mathrm{g} / \mathrm{mL}$ puromycin twice before inoculation. The male 6-weeks SCID mice were anesthetized, the muscles of the abdomen area were cut after retracting the skin, and the prostate gland exposed. $50 \mu \mathrm{L}$ of PC-3-Luc cell suspension at $0.5 \times 10^{6} / \mathrm{mL}$ in Matrigel ${ }^{\circledR}$ was injected into a dorsal prostatic lobe. The wound was closed in two layers and the skin was clipped. Animals were given analgesic drugs for 3 days and the tumors were monitored by an IVIS optical imaging system.

Bioluminescent imaging. For in vitro imaging, PC-3-Luc cells (30,000 cells/well) were diluted and plated in a 96-well plate. D-luciferin $(50 \mu \mathrm{L}, 150 \mu \mathrm{g} / \mathrm{mL})$ was added to the media five min prior to imaging (Figure S1). For in vivo imaging, mice were given D-luciferin $(100 \mu \mathrm{L}, 15 \mathrm{mg} / \mathrm{mL}) 15-20 \mathrm{~min}$ prior to anesthetization by isoflurane. At imaging, the mice were transferred to the IVIS enclosure and images were acquired by IVIS ${ }^{\circledR}$ Spectrum software with auto-exposure. Regions of interest from each image were selected and quantified.

In Vivo biodistribution studies. The mice were injected, monitored and dissected in the lab with the temperature of $23^{\circ} \mathrm{C}$ and $17 \%$ relative humidity. All animals were awake until sacrifice. The body weight, tumor volume and tumor luminescence of the mice were recorded every three days. At four-to-seven weeks post-xenograft implantation, the mice were divided into three groups by tumor volume: $<300,300-700$ and $>700 \mathrm{~mm}^{3}$ in both animal models. Tumor volume groups of the subcutaneous models had an $\mathrm{N}=8\left(<300 \mathrm{~mm}^{3}\right), 9\left(300-700 \mathrm{~mm}^{3}\right)$ and $9\left(>700 \mathrm{~mm}^{3}\right)$ of mice separately. The orthotopic tumor volume groups had an $\mathrm{N}=9\left(<300 \mathrm{~mm}^{3}\right), 8(300-$ $700 \mathrm{~mm}^{3}$ ), and $6\left(>700 \mathrm{~mm}^{3}\right)$ of mice separately. Each mouse (average weight: $20 \mathrm{~g}$ for female mice and $25 \mathrm{~g}$ for male mice) was treated with pimonidazole solution $(80 \mathrm{mg} / \mathrm{kg}$ in PBS) via intraperitoneal injection. After $1 \mathrm{~h}$, the mice received an intravenous injection of $10 \mu \mathrm{Ci}(370 \mathrm{kBq})$ of the radio-RP-HPLC peak purified ${ }^{177} \mathrm{Lu}$-labeled conjugate $\left(\left[{ }^{177} \mathrm{Lu}\right] \mathrm{Lu}-\mathrm{DOTA}-\mathrm{SP} 714\right)$ in $100 \mu \mathrm{L}$ of PBS. After an additional $1 \mathrm{~h}$, the mice were injected intravenously with a PBS solution containing $10 \mu \mathrm{Ci}(370 \mathrm{kBq})$ of $\left[{ }^{99 \mathrm{~m} \mathrm{Tc}}\right] \mathrm{NaTcO} 4$ and $15 \mathrm{mg} / \mathrm{kg}$ Hoechst 33342 . The animals were sacrificed 5 minutes later, and their tissues collected. The excised tissues were weighed, the radioactivity for each tissue was measured by $\gamma$-counter and the percentage injected dose per gram $(\% \mathrm{ID} / \mathrm{g})$ was calculated for each tissue.

Microscopy and autoradiography. At the end of the biodistribution studies, the tumor, liver, kidney and pancreas from the mice were rinsed by deionized water, dried and embedded by O.C.T compound on dry ice. The adjacent cryostat tumor slides $(10 \mu \mathrm{m})$ were scanned for Hoechst 33342 and anti-pimonidazole-FITC by confocal microscopy and exposed to a storage phosphor screen to be scanned by the Typhoon imaging system using $25 \mu \mathrm{m}$ 
resolution. The fluorescent intensity of images was measured by ZEN software (blue edition). The H\&E staining sections of the liver, kidney and pancreas were evaluated by a clinical pathologist. Frozen slides have significant high background fluorescence caused by non-seal storage were excluded from the analysis.

Statistical analysis. Data were presented as Mean \pm SD/SEM as noted. IC50 values were determined by nonlinear regression using the one-binding-site model of GraphPad PRISM 5. Pearson correlation coefficients (r) were calculated to assess the relationship between tumor volume and tumor uptake of the radiotracer $\left(\left[{ }^{177} \mathrm{Lu}\right]\right.$ $\mathrm{Lu}-\mathrm{DOTA}-\mathrm{SP} 714$ and $\left.\left[{ }^{99 \mathrm{~m}} \mathrm{Tc}\right] \mathrm{NaTcO}_{4}\right)$. In vitro stability studies of the radioconjugate in various buffers were analyzed by the two-tailed Student's t-test. For comparison between the two models, analysis of the biodistribution studies, hypoxic burden and blood vessel density of tumor was carried out by a two-tailed Student's t-test. Comparisons of the means of the BB2r-uptake, blood perfusion, hypoxic burden and blood vessel density of the tumors among the three tumor volume groups for each animal model were analyzed by one-way ANOVA. If the overall p-value was statistically significant, pairwise comparisons were adjusted using Tukey's method. A p-value $<0.05$ was considered statistically significant.

Ethical approval. All animal experiments were performed in accordance with the NIH animal use guideline and protocol approved by the Institutional Animal Care and Use Committee (IACUC) at the University of Nebraska Medical Center. None human participants were involved in this study.

\section{Data Availability}

The datasets generated during and/or analyzed during the current study are available from the corresponding author on reasonable request.

\section{References}

1. Patel, O., Shulkes, A. \& Baldwin, G. S. Gastrin-releasing peptide and cancer. Biochim Biophys Acta 1766, 23-41 (2006).

2. Van de Wiele, C. et al. Biodistribution and dosimetry of ${ }^{99 m T c-R P 527, ~ a ~ g a s t r i n-r e l e a s i n g ~ p e p t i d e ~(G R P) ~ a g o n i s t ~ f o r ~ t h e ~ v i s u a l i z a t i o n ~}$ of GRP receptor-expressing malignancies. J Nucl Med 42, 1722-1727 (2001).

3. Van de Wiele, C. et al. Technetium-99m RP527, a GRP analogue for visualisation of GRP receptor-expressing malignancies: a feasibility study. Eur J Nucl Med 27, 1694-1699 (2000).

4. Minamimoto, R. et al. Pilot Comparison of ${ }^{68} \mathrm{Ga}-\mathrm{RM} 2$ PET and ${ }^{68} \mathrm{Ga}-\mathrm{PSMA}-11$ PET in Patients with Biochemically Recurrent Prostate Cancer. J Nucl Med 57, 557-562 (2016).

5. Zhang, J. et al. PET Using a GRPR Antagonist ${ }^{68}$ Ga-RM26 in Healthy Volunteers and Prostate Cancer Patients. J Nucl Med 59, 922-928 (2018).

6. Yang, M. et al. F-Labeled GRPR Agonists and Antagonists: A Comparative Study in Prostate Cancer Imaging. Theranostics 1, 220-229 (2011)

7. Wu, X., Gong, S., Roy-Burman, P., Lee, P. \& Culig, Z. Current mouse and cell models in prostate cancer research. Endocr Relat Cancer 20, R155-170 (2013).

8. Talmadge, J. E., Singh, R. K., Fidler, I. J. \& Raz, A. Murine models to evaluate novel and conventional therapeutic strategies for cancer. The American journal of pathology 170, 793-804 (2007).

9. Zhou, Z. et al. Synthesis and in vitro and in vivo evaluation of hypoxia-enhanced 111In-bombesin conjugates for prostate cancer imaging. J Nucl Med 54, 1605-1612 (2013).

10. Mansi, R. et al. Evaluation of a 1,4,7,10-tetraazacyclododecane-1,4,7,10-tetraacetic acid-conjugated bombesin-based radioantagonist for the labeling with single-photon emission computed tomography, positron emission tomography, and therapeutic radionuclides. Clin Cancer Res 15, 5240-5249 (2009).

11. Dalm, S. U. et al. ${ }^{68} \mathrm{Ga} /{ }^{177} \mathrm{Lu}-\mathrm{NeoBOMB} 1$, a Novel Radiolabeled GRPR Antagonist for Theranostic Use in Oncology. J Nucl Med 58, 293-299 (2017).

12. Levi, J., Sathirachinda, A. \& Gambhir, S. S. A high-affinity, high-stability photoacoustic agent for imaging gastrin-releasing peptide receptor in prostate cancer. Clin Cancer Res 20,3721-3729 (2014).

13. Zhang, X. et al. $18 \mathrm{~F}$-labeled bombesin analogs for targeting GRP receptor-expressing prostate cancer. J Nucl Med 47, $492-501$ (2006).

14. Fukumura, D. \& Jain, R. K. Tumor microvasculature and microenvironment: targets for anti-angiogenesis and normalization. Microvasc Res 74, 72-84 (2007).

15. Brown, J. M. Exploiting the hypoxic cancer cell: mechanisms and therapeutic strategies. Mol Med Today 6, 157-162 (2000).

16. Sooriakumaran, P. \& Kaba, R. Angiogenesis and the tumour hypoxia response in prostate cancer: a review. Int J Surg 3, 61-67 (2005).

17. Burke, G. \& Halko, A. Cerebral blood flow studies with sodium pertechnetate Tc 99m and the scintillation camera. JAMA 204, 319-324 (1968).

18. Pavlov-Dolijanovic, S. et al. Diagnosis of Raynaud's phenomenon by ${ }^{99 m}$ Tc-pertechnetate hand perfusion scintigraphy: a pilot study. Rheumatology international 36, 1683-1688 (2016).

19. Salimi, Z. et al. Assessment of tissue viability in frostbite by ${ }^{99 m}$ Tc pertechnetate scintigraphy. AJR Am J Roentgenol 142, $415-419$ (1984).

20. Bhatnagar, A. et al. Diagnosis, characterisation and evaluation of treatment response of frostbite using pertechnetate scintigraphy: a prospective study. Eur J Nucl Med Mol Imaging 29, 170-175 (2002).

21. Sathekge, M. M., Mageza, R. B., Muthuphei, M. N., Modiba, M. C. \& Clauss, R. C. Evaluation of thyroid nodules with technetium99m MIBI and technetium-99m pertechnetate. Head Neck 23, 305-310 (2001).

22. Suonio, S. \& Olkkonen, H. A method for the assessment of placental blood perfusion using 99mTc pertechnetate. Scand J Clin Lab Invest 37, 509-515 (1977).

23. Trotter, M. J., Olive, P. L. \& Chaplin, D. J. Effect of vascular marker Hoechst 33342 on tumour perfusion and cardiovascular function in the mouse. Br J Cancer 62, 903-908 (1990).

24. Varia, M. A. et al. Pimonidazole: a novel hypoxia marker for complementary study of tumor hypoxia and cell proliferation in cervical carcinoma. Gynecol Oncol 71, 270-277 (1998).

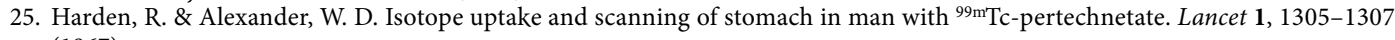
(1967).

26. Pavese, J., Ogden, I. M. \& Bergan, R. C. An orthotopic murine model of human prostate cancer metastasis. J Vis Exp, e50873, (2013).

27. Garrison, W. M. Reaction mechanisms in the radiolysis of peptides, polypeptides, and proteins. Chemical Reviews 87, 381-398 (1987). 
28. Rao, M. \& Rao, M. N. Protective effects of selenomethionine against cisplatin-induced renal toxicity in mice and rats. J Pharm Pharmacol 50, 687-691 (1998).

29. Rostami, A., Moosavi, S. A., Changizi, V. \& Abbasian Ardakani, A. Radioprotective effects of selenium and vitamin-E against 6MV X-rays in human blood lymphocytes by micronucleus assay. Med J Islam Repub Iran 30, 367 (2016).

30. Bakir, M. A., Alya, G., Mohammad, A., Azroony, R. \& Kasies, F. Radio-protective effects of selenium in rats. Journal of Radioanalytical and Nuclear Chemistry 266, 165-170 (2005).

31. Chen, J. et al. Synthesis, stabilization and formulation of $\left[{ }^{177} \mathrm{Lu}\right] \mathrm{Lu}-\mathrm{AMBA}$, a systemic radiotherapeutic agent for Gastrin Releasing Peptide receptor positive tumors. Appl Radiat Isot 66, 497-505 (2008).

32. Sato, N. et al. A metastatic and androgen-sensitive human prostate cancer model using intraprostatic inoculation of LNCaP cells in SCID mice. Cancer Res 57, 1584-1589 (1997).

33. Burrell, J. S. et al. Investigating the Vascular Phenotype of Subcutaneously and Orthotopically Propagated PC3 Prostate Cancer Xenografts Using Combined Carbogen Ultrasmall Superparamagnetic Iron Oxide MRI. Top Magn Reson Imaging 25, 237-243 (2016).

34. Ho, K. S., Poon, P. C., Owen, S. C. \& Shoichet, M. S. Blood vessel hyperpermeability and pathophysiology in human tumour xenograft models of breast cancer: a comparison of ectopic and orthotopic tumours. BMC cancer 12, 579 (2012).

35. Penet, M. F. et al. Noninvasive multiparametric imaging of metastasis-permissive microenvironments in a human prostate cancer xenograft. Cancer Res 69, 8822-8829 (2009).

36. Forster, J. C., Harriss-Phillips, W. M., Douglass, M. J. \& Bezak, E. A review of the development of tumor vasculature and its effects on the tumor microenvironment. Hypoxia 5, 21-32 (2017)

37. Chang, Y. S. et al. Mosaic blood vessels in tumors: frequency of cancer cells in contact with flowing blood. Proc Natl Acad Sci USA 97, 14608-14613 (2000).

38. Marignol, L., Coffey, M., Lawler, M. \& Hollywood, D. Hypoxia in prostate cancer: a powerful shield against tumour destruction? Cancer treatment reviews 34, 313-327 (2008).

39. Schroeder, R. P. et al. A standardised study to compare prostate cancer targeting efficacy of five radiolabelled bombesin analogues. Eur J Nucl Med Mol Imaging 37, 1386-1396 (2010).

40. Osieka, R., Houchens, D. P., Goldin, A. \& Johnson, R. K. Chemotherapy of human colon cancer xenografts in athymic nude mice. Cancer 40, 2640-2650 (1977).

41. Milross, C. G. et al. The effect of tumor size on necrosis and polarographically measured pO2. Acta Oncol 36, 183-189 (1997)

42. Euhus, D. M., Hudd, C., LaRegina, M. C. \& Johnson, F. E. Tumor measurement in the nude mouse. J Surg Oncol 31, 229-234 (1986).

43. Burgi, S. et al. In vivo imaging of hypoxia-inducible factor regulation in a subcutaneous and orthotopic GL261 glioma tumor model using a reporter gene assay. Mol Imaging 13 (2014).

44. Graves, E. E. et al. Hypoxia in models of lung cancer: implications for targeted therapeutics. Clin Cancer Res 16, 4843-4852 (2010).

45. Senapati, S. et al. Overexpression of macrophage inhibitory cytokine-1 induces metastasis of human prostate cancer cells through the FAK-RhoA signaling pathway. Oncogene 29, 1293-1302 (2010).

\section{Acknowledgements}

We thank Janice A. Taylor and James R. Talaska of the Advanced Microscopy Core Facility at the University of Nebraska Medical Center (UNMC) for providing assistance with confocal microscopy. We also thank Jiang Jiang and Lijun Sun of the Tissue Science Facility at UNMC for providing assistance with H\&E staining. This study was funded by the National Institutes of Health (5R01CA179059-04).

\section{Author Contributions}

W.Z. and J.C.G. designed the experiments and wrote the manuscript. All authors participated in conducting the experiments. W.Z., Z.Z. and J.C.G. analyzed the results. All authors reviewed the manuscript.

\section{Additional Information}

Supplementary information accompanies this paper at https://doi.org/10.1038/s41598-019-47308-z.

Competing Interests: The authors declare no competing interests.

Publisher's note: Springer Nature remains neutral with regard to jurisdictional claims in published maps and institutional affiliations.

(i) Open Access This article is licensed under a Creative Commons Attribution 4.0 International

License, which permits use, sharing, adaptation, distribution and reproduction in any medium or format, as long as you give appropriate credit to the original author(s) and the source, provide a link to the Creative Commons license, and indicate if changes were made. The images or other third party material in this article are included in the article's Creative Commons license, unless indicated otherwise in a credit line to the material. If material is not included in the article's Creative Commons license and your intended use is not permitted by statutory regulation or exceeds the permitted use, you will need to obtain permission directly from the copyright holder. To view a copy of this license, visit http://creativecommons.org/licenses/by/4.0/.

(C) The Author(s) 2019 